\title{
Enumerative Geometry of Divisorial Families of Rational Curves
}

\author{
ZIV RAN
}

\begin{abstract}
We compute the number of irreducible rational curves of given degree with 1 tacnode in $\mathbb{P}^{2}$ or 1 node in $\mathbb{P}^{3}$ meeting an appropriate generic collection of points and lines. As a byproduct, we also compute the number of rational plane curves of degree $d$ passing through $3 d-2$ given points and tangent to a given line. The method is 'classical', free of Quantum Cohomology.
\end{abstract}

Mathematics Subject Classification (2000): 14H50 (primary); 14N10 (secondary).

In the past 15 years or so a number of classical problems in the enumerative geometry of curves in $\mathbb{P}^{n}$ were solved, first for $n=2$, any genus [R1], then for any $n$, genus 0 . The latter development was initiated by Kontsevich-Manin who developed and used the rather substantial machinery of Quantum Cohomology (cf. e.g. [FP]). Subsequently, in a series of papers [R2]-[R5] the author developed an elementary alternative approach, free of Quantum cohomology, and used it to solve a number of classical enumerative problems for rational, and sometimes elliptic, curves in $\mathbb{P}^{n}, n \geq 2$. The present paper continues this series. The object here is to enumerate the irreducible rational curves of given degree $d$ in $\mathbb{P}^{2}$ with one tacnode passing through $3 d-2$ general points (see Theorems 2 and 5 below), as well as the irreducible rational curves in $\mathbb{P}^{3}$ with one ordinary node which contain $a$ general points and are incident to $4 d-2 a-1$ general lines (see Theorem 1 below). Note that the family of 1-tacnodal (resp. 1-nodal) curves in $\mathbb{P}^{2}$ (resp. $\mathbb{P}^{3}$ ) is of codimension 1 in the family of all rational curves, so that we are effectively computing the 'degree', in a sense, of certain natural divisors in the family of all rational curves. Indeed by a result of Diaz and Harris $[\mathrm{DH}]$ in the case of $\mathbb{P}^{2}$, the general member of any such divisor, if not nodal, is either 1-cuspidal (which case was enumerated in $[\mathrm{P}],[\mathrm{R} 2]$ ) or 1-tacnodal or has 1 triple point (which case is enumerated in [R6]). It seems very likely, but does not seem to be in the literature, that the natural analogue

Research Partially supported by NSA Grant MDA904-02-1-0094. Duplication and distribution by US Government permitted.

Pervenuto alla Redazione il 28 aprile 2003 ed in forma definitiva il 5 febbraio 2004. 
of this result also holds for any $n \geq 3$ : i.e. that the general member of any divisor in the family of rational curves is either smooth, or 1-nodal reducible (any $n \geq 3$ ), or, if $n=3,1$-nodal irreducible. As a byproduct of the proof of Theorem 2, we also obtain a formula for one of the 'characteristic numbers' for rational plane curves, viz. the number of such curves of degree $d$ passing through $3 d-2$ general points and tangent to a given line (cf. Corollary 4 below).

Enumeration of rational plane curves with a tacnode or triple point appears to be well within the range of interest, at least, of classical geometers. For quartics, very far-reaching results of this kind, including all the characteristic numbers for quartics with one cusp, one tacnode or one triple point, were obtained by Zeuthen [Z]. Recently, enumerative results for curves with 'few' singularities on general surfaces were obtained by Kleiman and Piene [KP]. Enumeration of 1-nodal rational curves in $\mathbb{P}^{n}$ for any $n$ was recently announced by Zinger [Zi], using Quantum Cohomology. As we shall see below, the 1 -nodal case in $\mathbb{P}^{3}$ case is analogous to, but easier than the 1-tacnodal case in $\mathbb{P}^{2}$. Our proof is based on the intersection calculus on the nonsingular model of the surface swept out by the appropriate 1-parameter family of rational curves, developed in earlier papers [R2]-[R4], together with elementary residual-intersection (in particular, double-point) theory as in [F].

We begin by reviewing some qualitative results about families of rational curves in $\mathbb{P}^{n}$, especially for $n=2$ or 3 . See [R2]-[R4], the Appendix to [R6], and references therein for details and proofs. In what follows we denote by $\bar{V}_{d}$ the closure in the Chow variety of the locus of irreducible nonsingular rational curves of degree $d$ in $\mathbb{P}^{n}, n=3$, with the scheme structure as closure, i.e. the reduced structure (recall that the Chow form of a reduced 1-cycle $Z$ is just the hypersurface in $G\left(1, \mathbb{P}^{3}\right)$ consisting of all linear spaces meeting $Z$ ); if $n=2$ $\bar{V}_{d}$ is just the (closure of) the Severi variety. Thus $\bar{V}_{d}$ is irreducible reduced of dimension

$$
\begin{gathered}
\operatorname{dim}\left(\bar{V}_{d}\right)=4 d, n=3 \\
\operatorname{dim}\left(\bar{V}_{d}\right)=3 d-1, n=2 .
\end{gathered}
$$

Let

$$
A_{1}, \ldots, A_{k} \subset \mathbb{P}^{n}
$$

be a generic collection of linear subspaces of respective codimensions $a_{1}, \ldots, a_{k}$, $2 \leq a_{i} \leq n$ (so if $n=2$ these are just points). We denote by

$$
B=B_{d}=B_{d}(a .)=B_{d}(A .)
$$

the normalization of the locus (with reduced structure)

$$
\left\{\left(C, P_{1}, \ldots, P_{k}\right): C \in \bar{V}_{d}, P_{i} \in C \cap A_{i}, i=1, \ldots, k\right\}
$$

which is also the normalization of its projection to $\bar{V}_{d}$, i.e. the locus of degree- $d$ rational curves (and their specializations) meeting $A_{1}, \ldots, A_{k}$. We have

$$
\operatorname{dim} B=(n+1) d+n-3-\sum\left(a_{i}-1\right) .
$$


When $\operatorname{dim} B=0$ we set

$$
N_{d}(a .)=\operatorname{deg}(B)
$$

When $n=2$, so all the $a_{i}=2$, they will be dropped. The integer $k$ is called the length of the condition-vector $\left(a\right.$.). The numbers $N_{d}$ and $N_{d}(a$.), first computed in general by Kontsevich and Manin (see for instance [FP] and references therein), are computed in [R2], [R3] by an elementary method based on recursion on $d$ and $k$.

Now suppose $\operatorname{dim} B=1$ and let

$$
\pi: X \rightarrow B
$$

be the normalization of the tautological family of rational curves, and

$$
f: X \rightarrow \mathbb{P}^{n}
$$

the natural map. The following summarizes results from [R2]-[R4]:

THEOREM 0.

(i) $X$ is smooth.

(ii) Each fibre $C$ of $\pi$ is either

(a) a $\mathbb{P}^{1}$ on which $f$ is either an immersion with at most one exception which maps to a cusp $(n=2)$ or an embedding $(n>2)$; or

(b) a pair of $\mathbb{P}^{1}$ 's meeting transversely once, on which $f$ is an immersion with nodal image $(n=2)$ or an embedding $(n>2)$; or

(c) if $n=3$, a $\mathbb{P}^{1}$ on which $f$ is a degree-1 immersion such that $f\left(\mathbb{P}^{1}\right)$ has a unique singular point which is an ordinary node.

(iii) If $n>2$ then $\bar{V}_{d, n}$ is smooth along the image $\bar{B}$ of $B$, and $\bar{B}$ is smooth except, in case some $a_{i}=2$, for ordinary nodes corresponding to curves meeting some $A_{i}$ of codimension 2 twice. If $n=2$ then $\bar{V}_{d, n}$ is smooth in codimension 1 except for a cusp along the cuspidal locus and normal crossings along the reducible locus, and $\bar{B}$ has the singularities induced from $\bar{V}_{d, n}$ plus ordinary nodes corresponding to curves with a node at some $A_{i}$, and no other singularities.

Next, we review some of the enumerative apparatus introduced in [R3], [R4] to study $X / B$. Let $s_{i}$ be the section of $X / B$ corresponding to $A_{i}$, i.e. the unique 1-dimensional component of $f^{-1}\left(A_{i}\right)$. Set

$$
m_{i}=m_{i}(a .)=-s_{i}^{2}, i=1, \ldots, k .
$$

Note that if $a_{i}=a_{j}$ then $m_{i}=m_{j}$; in particular for $n=2$ they are all equal and will be denoted by $m_{d}$. It is shown in [R2]-[R4] that these numbers can all be computed recursively in terms of data of lower degree $d$ and lower length $k$. For instance for $n=2$ we have (cf. [R2], display (1.8) page 113)

$$
2 m_{d}=\sum_{d_{1}+d_{2}=d} N_{d_{1}} N_{d_{2}} d_{1} d_{2}\left(\begin{array}{c}
3 d-4 \\
3 d_{1}-2
\end{array}\right) \text {. }
$$


For $n>2$, note that

$$
s_{i} . s_{j}=N_{d}\left(\ldots, a_{i}+a_{j}, \ldots, \hat{a}_{j}, \ldots\right), i \neq j .
$$

(so for $n=2$ this is always 0 ). Also, letting $R_{i}$ denote the sum of all fibre components not meeting $s_{i}$, we have

$$
s_{1} \cdot R_{2}=\sum N_{d_{1}}\left(A_{.}^{1}, A_{1}, \mathbb{P}^{s_{1}}\right) N_{d_{2}}\left(A^{2}, A_{2}, \mathbb{P}^{s_{2}}\right) .
$$

the summations being over all $d_{1}+d_{2}=d, s_{1}+s_{2}=3$ and all decompositions $A .=\left(A_{1}, A_{2}\right) \amalg\left(A^{1}\right) \amalg\left(A^{2}\right)$ (as unordered sequences or partitions); similarly for the other $s_{i} . R_{j}$. So all these numbers may be considered known. Then we have

$$
m_{i}=\frac{1}{2}\left(s_{i} \cdot R_{j}+s_{i} \cdot R_{p}-s_{j} \cdot R_{p}\right)-s_{i} \cdot s_{j}-s_{i} \cdot s_{p}+s_{j} \cdot s_{p}
$$

for any distinct $i, j, p$, and the RHS here is an expression of lower degree and/or length, hence may be considered known.

Next, set

$$
L=f^{*}(\mathcal{O}(1))
$$

and note that

$$
L^{2}=N_{d}(2, a .), \quad L . s_{i}=N_{d}\left(a_{1}, \ldots, a_{i+1} \ldots\right), i=1, \ldots, k
$$

(in particular, L. $s_{i}=0$ if $a_{i}=n$.) We computed in [R3] that, for any $i$,

$$
L \sim d s_{i}-\sum_{F \in \mathcal{F}_{i}} \operatorname{deg}(F) F+\left(N_{d}\left(a_{1}, \ldots, a_{i}+1, \ldots\right)+d m_{i}\right) F_{0}
$$

where $F_{0}$ is the class of a complete fibre and $\mathcal{F}_{i}$ is the set of fibre components not meeting $s_{i}$. Consequently we have

$$
N_{d}\left(2, a_{1}, \ldots\right)=2 d N_{d}\left(a_{1}+1, a_{2}, \ldots\right)+d^{2} m_{1}(a .)-\sum_{F \in \mathcal{F}_{1}(a .)}(\operatorname{deg} F)^{2}
$$

and clearly the RHS is a lower degree/length expression, so all the $N_{d}(2, \ldots)$ are known. We also have for $n>2$ that

$$
\begin{aligned}
& N_{d}\left(a_{1}, a_{2}+1, \ldots\right)-N_{d}\left(a_{1}+1, a_{2}, \ldots\right) \\
& =d N_{d}\left(a_{1}+a_{2}, \ldots\right)-\sum_{F \in\left(\mathcal{F}_{1}-\mathcal{F}_{2}\right)(a .)}(\operatorname{deg} F)+N_{d}\left(a_{1}+1, a_{2}, \ldots\right)+d m_{1}(a .)
\end{aligned}
$$

and again the RHS here is 'known', hence so is the LHS, which allows us to 'shift weight' between the $a_{i}$ 's till one of them becomes 2, so we may apply (7), and thus compute all of the $N_{d}(a$.)'s. 
Next, it is easy to see as in [R3] that

$$
L . R_{i}=\sum_{d_{1}+d_{2}=d}\left(\begin{array}{c}
3 d-1 \\
3 d_{1}-1
\end{array}\right) d_{1} d_{2}^{2} N_{d_{1}} N_{d_{2}}, \quad n=2
$$

(in this case this is independent of $i$ and we will just write it as $L . R$ );

$$
L . R_{i}=\sum d_{2} N_{d_{1}}\left(a^{1}, s_{1}\right) N_{d_{2}}\left(a^{2}, s_{2}\right), n>2
$$

the summation for $n>2$ being over all $d_{1}+d_{2}=d, s_{1}+s_{2}=3$ and all decompositions

$$
\text { A. }=\left(A_{.}^{1}\right) \coprod\left(A_{.}^{2}\right)
$$

(as unordered sequences or partitions) such that $A_{i} \in\left(A^{1}\right.$ ).

Finally, the relative canonical class $K_{X / B}=K_{X}-\pi^{*}\left(K_{B}\right)$, which we will denote by $K$, was computed in [R3] as

$$
K_{X / B}=-2 s_{i}-m_{i} F+R_{i}
$$

for any $i$. Note that $-R_{i}^{2}$ equals the number of reducible fibres in the family $X / B$, a number we denote by $N_{d}^{\text {red }}(a)$, and which is easily computable by recursion. From this we compute that

$$
L . K_{X / B}=-2 N_{d}\left(\ldots a_{i}+1 \ldots\right)-d m_{i}+L \cdot R_{i} .
$$

For $n=2$ the first term in (12) vanishes, so in view of (5), (9) we get

$$
\begin{gathered}
L . K_{X / B}=\sum_{d_{1}+d_{2}=d}\left(\begin{array}{c}
3 d-1 \\
3 d_{1}-1
\end{array}\right) d_{1} d_{2}^{2} N_{d_{1}} N_{d_{2}}-\frac{d}{2} \sum_{d_{1}+d_{2}=d} N_{d_{1}} N_{d_{2}} d_{1} d_{2}\left(\begin{array}{c}
3 d-4 \\
3 d_{1}-2
\end{array}\right) \\
n=2 .
\end{gathered}
$$

Also,

$$
K_{X / B}^{2}=-N_{d}^{\mathrm{red}}\left(a_{.}\right) .
$$

For $n=2$ we denote by $\kappa_{d}$ the number of rational curves with a cusp passing through $3 d-2$ generic points in $\mathbb{P}^{2}$, a number that was computed in [R3] (see the display on $1 .-6$, p.11) as

$$
\kappa_{d}=3\left(N_{d}+L . K\right)-2 N_{d}^{\mathrm{red}} .
$$

Now in case $n=3$, for any condition-vector (a.) of weight

$$
\sum\left(a_{i}-1\right)=4 d-1
$$

we denote by $N_{d}^{\times}(a$.) the number of 1-nodal irreducible rational curves in $P^{3}$ meeting a generic collection of linear spaces of respective codimensions $\left(a_{1}, \ldots, a_{k}\right)$. 
THEOREM 1. We have for any $i=1, \ldots, k$,

$$
N_{d}^{\times}(a)=(d-2) N_{d}\left(2, a_{.}\right)-4 N_{d}\left(\ldots a_{i}+1 \ldots\right)-2 d m_{i}+2 L . R_{i}+N_{d}^{\mathrm{red}}(a .)
$$

ProOF. We set things up so as to apply a relative double point formula from [R6]. Let

$$
X_{B}^{2}
$$

denote the fibre square of $X / B$, and

$$
\Delta \simeq X \subset X_{B}^{2}
$$

the diagonal. Then $X_{B}^{2}$ is smooth except at points $(p, p)$, where $p$ is a singular point of a fibre of $X / B$. Locally at such points, if $X / B$ is given locally at $p$ by

$$
x y=t,
$$

then $X_{B}^{2}$ is given by

$$
x_{1} y_{1}=x_{2} y_{2}=t,
$$

and therefore has an ordinary 3 -fold double point. Moreover the diagonal $\Delta$ is defined by

$$
x_{1}=x_{2}, y_{1}=y_{2}
$$

and in particular is a non-Cartier divisor. Let

$$
b: Y \rightarrow X_{B}^{2}
$$

denote the blow-up of $\Delta$, with exceptional divisor

$$
\Delta^{\prime}=b^{*}(\Delta)
$$

Thus $Y$ is the subvariety of $X_{B}^{2} \times \mathbb{P}^{1}$ defined by the equation

$$
U_{0}\left(y_{2}-y_{1}\right)=U_{1}\left(x_{2}-x_{1}\right)
$$

where $U_{0}, U_{1}$ are homogeneous coordinates on $\mathbb{P}^{1}$. In other words, $Y$ is simply the graph of the rational function (or rational map to $\mathbb{P}^{1}$ ) given by $\frac{y_{2}-y_{1}}{x_{2}-x_{1}}$ As usual, $Y$ is covered by 2 opens on each of which either $\frac{y_{2}-y_{1}}{x_{2}-x_{1}}$ or $\frac{x_{2}-x_{1}}{y_{2}-y_{1}}$ is a regular function. Then it is easy to see that $b$ is a small resolution of $X_{B}^{2}$ with exceptional locus

$$
E=\sum E_{p}
$$

consisting of a $\mathbb{P}^{1}$ for each relatively singular point $p$. Moreover the fibre of $Y$ over $B$ corresponding to a reducible fibre $C_{1} \cup_{p} C_{2}$ of $X / B$ is of a 'honeycomb' shape

$$
\begin{array}{lll}
B_{(p, p)} C_{1}^{2} & \cup & C_{1} \times C_{2} \\
C_{2} \times C_{1} & \cup & B_{(p, p)} C_{2}^{2}
\end{array}
$$


where

$$
B_{(p, p)} C_{1}^{2} \cap B_{(p, p)} C_{2}^{2}=E_{p}, C_{1} \times C_{2} \cap C_{2} \times C_{1}=\emptyset .
$$

Here we use the notation $B_{x} Y$ to denote the blowup of a variety $Y$ in a subvariety (or point) $x$. Furthermore, the identity of rational functions, locally at $p$,

$$
\frac{y_{2}-y_{1}}{x_{2}-x_{1}}=\frac{y_{1}}{x_{2}}=\frac{y_{2}}{x_{1}}
$$

shows that there $b: Y \rightarrow X_{B}^{2}$ coincides with the blowup of the ideal $\left(y_{1}, x_{2}\right)$, and with that of the ideal $\left(y_{2}, x_{1}\right)$ (since all these blowups are the graph of the same rational function). This implies that $\Delta^{\prime} \rightarrow \Delta$ is just the blowing up of all the points $p$.

We will need to know the normal bundle

$$
v=N_{\Delta^{\prime} / Y}
$$

To this end, note that, identifying $\Delta$ with $X$ as above, the map $\Delta^{\prime} \rightarrow X$ is a blowup of smooth points, hence

$$
\omega_{\Delta^{\prime}}=b^{*} \omega_{X}(E)
$$

On the other hand, the map (15) is small, hence crepant, so

$$
\omega_{Y}=b^{*} \omega_{X_{B}^{2}}
$$

Now $X_{B}^{2}$ as divisor on $X^{2}$ is the pullback of the diagonal divisor $\Delta_{B} \subset B^{2}$, whose normal bundle is $\omega_{B}^{-1}$. By adjunction, it follows that

$$
\omega_{X_{B}^{2}}=p_{1}^{*} \omega_{X} \otimes p_{2}^{*} \omega_{X} \otimes \pi^{*} \omega_{B}^{-1}
$$

and consequently

$$
\omega_{Y}=b_{1}^{*} \omega_{X} \otimes b_{2}^{*} \omega_{X} \otimes c^{*} \omega_{B}^{-1}
$$

where we use the evident maps

$$
b_{i}: Y \rightarrow X, i=1,2, c: Y \rightarrow B
$$

Therefore by adjunction again we conclude

$$
v=b^{*} \omega_{X / B}^{-1}(E) \text {. }
$$

Now it is shown in [R6], and is in any case not hard to see, that $Y$ admits a natural map to the Hilbert scheme of $X$, in fact to $B_{\Delta_{X}}(X \times X)$, which dominates the Hilbert scheme and therefore $Y$ can be used to enumerate double 
points. Specifically, the double-point formula of [R6, Corollary 3.4] uses the natural map

$$
f^{2}=\left(f_{1}, f_{2}\right): Y \rightarrow \mathbb{P}^{3} \times \mathbb{P}^{3} .
$$

and shows that the cycle of points in $Y$ corresponding to ordered pairs $\left(x_{1}, x_{2}\right)$ in $X / B^{2}$ such that $f\left(x_{1}\right)=f\left(x_{2}\right)$ is residual to $\Delta^{\prime}$ in $f^{2 *}\left(\Delta_{\mathbb{P}^{3}}\right)$ and is thus a 0 -scheme of the rank-3 bundle on $Y$

$$
f_{1}^{*}(\mathcal{O}(1)) \otimes f_{2}^{*}(Q) \otimes \mathcal{O}\left(-\Delta^{\prime}\right),
$$

where $Q$ denotes the universal quotient bundle on $\mathbb{P}^{3}$ and $p_{1}^{*} \mathcal{O}(1) \otimes p_{2}^{*} Q$ appears as a 'grassmannian bundle', i.e. a bundle on $\mathbb{P}^{3} \times \mathbb{P}^{3}$ having the diagonal as zero-set.

Therefore we find that

$$
N_{d}^{\times}(a .)=\frac{1}{2} c_{3}\left(f_{1}^{*}(\mathcal{O}(1)) \otimes f_{2}^{*}(Q) \otimes \mathcal{O}\left(-\Delta^{\prime}\right)\right) .
$$

Then a straightforward calculation, based on the intersection calculus reviewed above, yields the formula stated in Theorem 1.

REMARK 1.1. Rather than appeal to a relative double-point formula, we could have used the usual double-point formula applied to the map

$$
f \times \pi: X \rightarrow \mathbb{P}^{3} \times B .
$$

This alternative approach is available generally, and will be explained in more detail in the course of the proof of Theorem 2.

We turn next to the enumeration of rational plane curves with a tacnode. Denote by $N_{d}^{\text {tac }}$ the number of rational curves with a tacnode passing through $3 d-2$ generic points in $\mathbb{P}^{2}$.

THEOREM 2. We have

$$
N_{d}^{\mathrm{tac}}=(3 d-11) N_{d}+(d-6) L K+4 N_{d}^{\mathrm{red}} .
$$

Proof. We use an analogous setup and notation as above, this time for a pencil of rational curves in $\mathbb{P}^{2}$ through $3 d-2$ points. To begin with, we describe an algebraic setup for the (relative) Gauss mapping. Consider the relative principal parts sheaf for $L$ on $X / B$, which fits in an exact sequence

$$
0 \rightarrow \Omega_{X / B}(L) \rightarrow P_{X / B}(L) \rightarrow L \rightarrow 0 .
$$

Setting $V=H^{0}\left(\mathcal{O}_{\mathbb{P}^{2}}(1)\right)$, we have a natural map $V \rightarrow P_{X / B}(L)$ which combines with the Euler sequence to yield an exact diagram

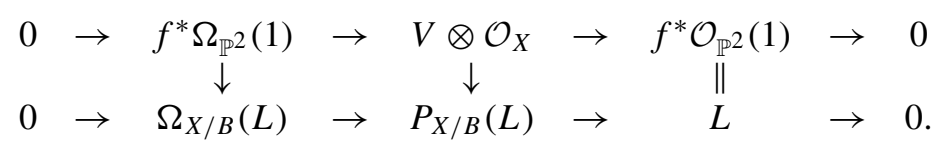


Let $P^{\prime}$ denote the pushout of $P_{X / B}(L)$ by the natural map

$$
\Omega_{X / B}(L) \rightarrow \omega_{X / B}(L) .
$$

LEMMA 3. The image of the composite map

$$
f^{*} \Omega_{\mathbb{P}^{2}} \rightarrow \Omega_{X / B} \rightarrow \omega_{X / B}
$$

coincides with $J \omega_{X / B}$ where $J$ is the ideal in $X$ of the fibre nodes and the 'cuspidal' points i.e. points $x$ such that $f(x)$ is a cusp of $f\left(\pi^{-1}(x)\right)$.

PROOF. Our map is obviously surjective at points that are neither cuspidal nor fibre nodes. Consider then a fibre node $p$. In local coordinates at $p$, the family $X / B$ is given by

$$
x y=t
$$

with $f=(x, y)$. Since

$$
\omega_{X / B}=\operatorname{Hom}\left(\pi^{*} \omega_{B}, \omega_{X}\right)
$$

and $\omega_{B}, \omega_{X}$ are generated respectively by $d t, d x \wedge d y, \omega_{X / B}$ is generated by $d x \wedge d y \cdot d t^{-1}$ (i.e the unique homomorphism taking $d t$ to $d x \wedge d y$ ). Moreover the natural map

$$
\Omega_{X / B} \rightarrow \omega_{X / B}
$$

takes a 1 -form $\alpha$ to the homomrphism

$$
(g d t \mapsto g d t \wedge \alpha) \in \omega_{X / B} .
$$

Since

$$
d x \wedge d t=x d x \wedge d y, d y \wedge d t=-y d x \wedge d y,
$$

we have that the image of $f^{*} \Omega_{\mathbb{P}^{2}}$ is generated by $x d x \wedge d y \cdot d t^{-1}, y d x \wedge d y \cdot d t^{-1}$, as claimed.

Consider now the cuspidal case. There the tautological family is locally universal over $\bar{B}$ by $[\mathrm{DH}]$, hence is locally given by an equation

$$
y^{2}=x^{3}+b_{1} x+b_{2}
$$

where $\left(b_{1}, b_{2}\right)$ are coordinates on $\bar{B}$ and satisfy

$$
27 b_{1}^{3}=8 b_{2}^{2} \text {. }
$$

The normalization of this local family is smooth, with coordinates $(u, t), t$ being a coordinate on $B$, and with normalization map given by

$$
\begin{gathered}
x=u^{2}-\frac{2}{3} t, \\
y=u\left(u^{2}-t\right), \\
\left(b_{1}, b_{2}\right)=\left(\frac{1}{3} t^{2}, \frac{2}{27} t^{3}\right) .
\end{gathered}
$$


Thus

$$
f=\left(u^{2}-\frac{2}{3} t,\left(u^{2}-t\right) u\right)=(x, y) .
$$

Now $\omega_{X / B}$ is generated by $d u$ with $d t$ going to 0 , and the image of the map from $f^{*} \Omega_{\mathbb{P}^{2}}$ is generated by

$$
d x=2 u d u, d y=\left(3 u^{2}-t\right) d u,
$$

hence clearly coincides with the subsheaf generated by $u d u$ and $t d u$, as claimed.

Now let $B_{1} \rightarrow B$ be any 2:1 cover whose branch locus contains the critical values, i.e. points $\pi(p)$ where $p$ is a fibre node, and which is unramified over the cuspidal values, i.e. the images of the cuspidal points. Then the induced map

$$
X_{B_{1}}:=X \times_{B} B_{1} \rightarrow X
$$

is ramified over the reducible fibres and $X_{B_{1}}$ has $A_{1}$ singularities over the fibre nodes. Let $X^{\prime} / B_{1}$ be the blowup of $X_{B_{1}}$ in those singularities, as well as in the preimages of the cuspidal points, with $E_{\sigma}$ and $E_{\kappa}$ the respective exceptional divisors and $E=E_{\sigma}+E_{\kappa}$,

$$
b: X^{\prime} \rightarrow X
$$

the natural map and $f^{\prime}=f \circ b$. It follows from Lemma 3 that if we let $P$ denote the image of the natural map

$$
V \otimes \mathcal{O}_{X^{\prime}} \rightarrow b^{*} P^{\prime}
$$

then we get a diagram with exact rows and columns

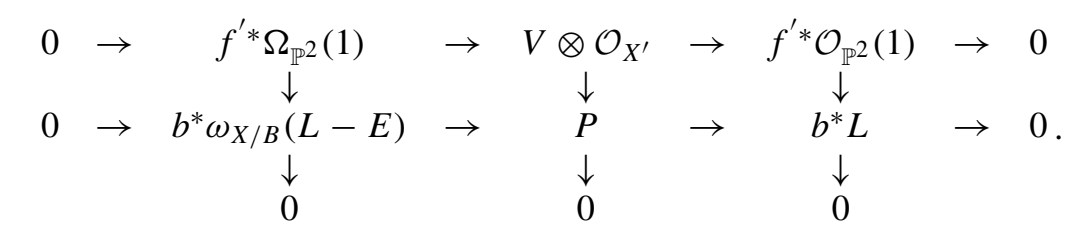

In the cuspidal case, note that by (21), the left vertical map in (22) is given by

$$
d x \mapsto 2 d u, d y \mapsto\left(2 u^{2}-t\right) d u
$$

Now let

$$
\phi: I \rightarrow \mathbb{P}^{2}
$$

be the incidence (or flag) variety, with universal flag

$$
\begin{aligned}
V \otimes \mathcal{O}_{I} & =F^{0} \supset F^{1}=\phi^{*} \Omega_{\mathbb{P}^{2}}(1) \supset F^{2} \supset(0), \\
F^{0} / F^{1} & =\phi^{*} \mathcal{O}(1) .
\end{aligned}
$$


Now the diagram (22) gives rise to a lifting of $f^{\prime}$ to a morphism

$$
g: X^{\prime} \rightarrow I
$$

with

$$
g^{*}\left(F^{0} / F^{1}\right)=\left.L\right|_{X^{\prime}}:=b^{*}(L), g^{*}\left(F^{1} / F^{2}\right)=b^{*}\left(\omega_{X / B}\right)(L-E)
$$

(we shall often use the same letter to denote a divisor and its pullbacks). Clearly, the value of $g$ at a point

$$
(x, b) \in X^{\prime} \backslash E
$$

mapping to

$$
y \in \mathbb{P}^{2}
$$

is the pair

$$
\left(y, T_{y}\left(f\left(X_{b}\right)\right)\right.
$$

consisting of $y$ and the tangent line to $f\left(X_{b}\right)$ at $f(y)$; in particular, each tacnode in the family $X / B$ yields 2 double points and 4 ordered double points of $g$, which we propose to count as in the proof of Theorem 1. Before proceeding it is convenient to note from (22) that the map $f^{v}: X^{\prime} \rightarrow \mathbb{P}^{2 v}$ obtained by composing $g$ with the natural projection of $I$ to the dual projective plane of lines in $\mathbb{P}^{2}$ corresponds to the invertible quotient (cf. (22))

$$
V^{v} \rightarrow V^{v} / P^{v} \rightarrow 0
$$

and it is clear from (22) that

$$
V^{v} / P^{v} \simeq \operatorname{det}(P) \simeq 2 L+K-E .
$$

The plan now is to apply the relative double-point formula as in the proof of Theorem 1 to the map $g: X^{\prime} \rightarrow I$ relative to $B_{1}$. Thus consider the fibre square

$$
\bar{Y}=X^{\prime} \times_{B_{1}} X^{\prime} .
$$

It is singular in its diagonal points corresponding to fibre nodes of $X^{\prime} / B_{1}$, and may be desingularized with small blowups as in the proof of Theorem 1. Let

$$
b_{Y}: Y \rightarrow \bar{Y}
$$

be the global desingularization thus obtained, and $\Delta^{\prime \prime} \subset Y$ the proper (=total) transform of the diagonal. It is easy to see that the map

$$
b^{\prime}: \Delta^{\prime \prime} \rightarrow X^{\prime}
$$

identifies $\Delta^{\prime \prime}$ with the blowup $X^{\prime \prime}$ of $X^{\prime}=\Delta^{\prime}$ in its own fibre nodes over $B_{1}$, i.e. the two intersection points of each component of $E_{\sigma}$ with the other components of its fibre, plus the one intersection point of each component of 
$E_{\kappa}$ with the other component of its fibre; call the new exceptional divisors thus obtained $E_{\sigma}^{\prime \prime}, E_{\kappa}^{\prime \prime}$ and set

$$
E^{\prime \prime}=E_{\sigma}^{\prime \prime}+E_{\kappa}^{\prime \prime}
$$

The normal bundle

$$
v=N_{\Delta^{\prime \prime} / Y}
$$

can be computed much like before: first,

$$
\omega_{\Delta^{\prime \prime}}=\left(b^{\prime}\right)^{*} \omega_{X^{\prime}}\left(E^{\prime \prime}\right)
$$

because $b^{\prime}$ blows up smooth points; second

$$
\left(b^{\prime}\right)^{*} \omega_{X^{\prime}}\left(E^{\prime \prime}\right)=\left(b^{\prime}\right)^{*} b^{*} \omega_{X}\left(E^{\prime \prime}\right)
$$

(because $b$ blows up $A_{1}$ singularities, hence does not change the canonical sheaf). As $Y \rightarrow \bar{Y}$ is a small blowup, it too does not change the canonical sheaf, hence as before,

$$
\omega_{Y}=\omega_{\bar{Y}}=p_{1}^{*} \omega_{X^{\prime}} \otimes p_{2}^{*} \omega_{X^{\prime}} \otimes \omega_{B_{1}}^{-1}
$$

therefore

$$
v=\omega_{\bar{Y}}^{-1} \omega_{\Delta^{\prime \prime}}=\omega_{X^{\prime}}^{-1} \omega_{B_{1}}\left(E^{\prime \prime}\right)=\left.\omega_{X_{1} / B_{1}}^{-1}\left(-E_{\kappa}+E^{\prime \prime}\right)\right|_{X^{\prime \prime}}=\left.\omega_{X / B}^{-1}\left(-E_{\kappa}+E^{\prime \prime}\right)\right|_{X^{\prime \prime}}
$$

the last equality because relative canonical sheaves commute with base change. Putting these together we see that, using divisor notation and setting $K=$ $K_{X}-K_{B}$,

$$
v=\left.\left(-K-E_{\kappa}+E_{\kappa}^{\prime \prime}+E_{\sigma}^{\prime \prime}\right)\right|_{X^{\prime \prime}}
$$

Consequently, we have

$$
v^{2}=2 K^{2}-2 N_{d}^{\mathrm{red}}-2 \kappa_{d}=-4 N_{d}^{\mathrm{red}}-2 \kappa_{d}
$$

where $\kappa_{d}$ is the number of cuspidal rational plane curves of degree $d$ through $3 d-2$ generic points, computed in [R3]. Of course, we also have

$$
L . v=-L \cdot X^{\prime \prime} K=-2 L \cdot{ }_{X} K=2(2 d m-L . R) .
$$

Now, we want to proceed as in the proof of Theorem 1 and use the relative double-point formula of [R6, Corollary 3.4]. To this end we need to show, in the terminology of [R6], that $I$ is 'pseudo-grassmannian', that is, we must produce a rank-3 vector bundle on $I \times I$ having the diagonal $\Delta_{I}$ as zero-scheme. To this end, consider the cartesian product $I^{2}=I \times I$ with projections

$$
p_{i}: I^{2} \rightarrow I, i=1,2, \phi^{2}: I^{2} \rightarrow\left(\mathbb{P}^{2}\right)^{2} .
$$


Let $F$. be the dual filtration to $F$, defined by

$$
F_{i}=\left(F^{0} / F^{i}\right)^{*} \subset\left(F^{0}\right)^{*} .
$$

In $I^{2}, \phi^{2 *} \Delta_{\mathbb{P}^{2}}$ is defined as the zero- locus of a map

$$
p_{1}^{*} F_{1} \rightarrow p_{2}^{*}\left(F_{3} / F_{1}\right)
$$

and inside this, $\Delta_{I}$ is defined as the zero- locus of a map

$$
p_{1}^{*}\left(F_{2} / F_{1}\right) \rightarrow p_{2}^{*}\left(F_{3} / F_{2}\right)
$$

Thus $\Delta_{I}$ is a zero scheme of a vector bundle $A$ on $I \times I$ where

$$
A=\left(\phi^{2}\right)^{-1}\left(A_{\mathbb{P}^{2}}\right) \oplus \mathcal{O}_{I \times I}(1,1) \otimes p_{1}^{*}(\operatorname{det} B)
$$

where $A_{\mathbb{P}^{2}}$ is a vector bundle on $\left(\mathbb{P}^{2}\right)^{2}$ with the diagonal as zero-scheme, e.g.

$$
p_{1}^{*}(\mathcal{O}(1)) \otimes p_{2}^{*} \Omega_{\mathbb{P}^{2}}(1),
$$

$B$ is a vector bundle on $\mathbb{P}^{2}$ with

$$
I=\mathbb{P}(B)
$$

(e.g. $B=\Omega_{\mathbb{P}^{2}}$ ), and

$$
\mathcal{O}_{I}(1)=\mathcal{O}_{\mathbb{P}(B)}(1)
$$

Considering as before the map

$$
g^{2}=\left(g_{1}, g_{2}\right): Y \rightarrow I^{2}
$$

double-point theory in the form of [ R6, Corollary 3.4], shows that the virtual number of ordered double points of $g$ (i.e. twice the virtual number of double points) is

$$
\begin{aligned}
& c_{3}\left(\left(g^{2}\right)^{*}(A)\left(-\Delta^{\prime \prime}\right)\right) \\
= & c_{2}\left(g_{1}^{*}\left(F_{1}^{*}\right) \otimes g_{2}^{*}\left(\left(V^{*} \otimes \mathcal{O}\right) / F_{1}\right) \otimes \mathcal{O}\left(-\Delta^{\prime \prime}\right)\right) c_{1}\left(g_{1}^{*}\left(\left(F_{2} / F_{1}\right)^{*}\right) \otimes g_{2}^{*}\left(F_{3} / F_{2}\right) \otimes \mathcal{O}\left(-\Delta^{\prime \prime}\right)\right) .
\end{aligned}
$$

Using (24), the $c_{2}$ factor can be identified, writing $L_{i}=p_{i}^{*} L, K_{i}=p_{i}^{*}\left(\omega_{X / B}\right)$, as

$$
\left.\left(L_{2}^{2}+L_{1} L_{2}-L_{2} \Delta^{\prime \prime}+\left(L_{1}-\Delta^{\prime \prime}\right)^{2}\right)\right|_{X^{\prime \prime}}
$$

while the $c_{1}$ factor is

$$
\left.\left(K_{1}+K_{2}+L_{1}+2 L_{2}-E_{1}-E_{2}-\Delta^{\prime \prime}\right)\right|_{X^{\prime \prime}} .
$$


Then a routine computation, using as above the necessary intersection theory on $Y$ and $X$ yields

$$
c_{3}\left(\left(g^{2}\right)^{*}(A)\left(-\Delta^{\prime \prime}\right)\right)=(4 d-24) K \cdot X+(12 d-32) N_{d}+2 N_{d}^{\mathrm{red}} .
$$

By the discussion above, this equals $4 N_{d}^{\text {tac }}$ plus the contribution from 'extraneous' double points of $g$ that do not come from tacnodes, i.e. that appear along the exceptional curves, and we shall analyze those below. Before doing so, however, we shall give another derivation of the formula (29), avoiding any explicit use of relative multiple point theory and using only the 'usual' double point formula of [F, Theorem 9.3].

The idea, which is valid more generally, is simply that relative (ordered) double points for a map such as $g$ with respect to $\pi: X^{\prime} \rightarrow B_{1}$ are the same as (ordered) double points in the usual (non-relative) sense for the product mapping

$$
h=g \times \pi: X^{\prime} \rightarrow I \times B_{1} .
$$

The number of ordered double points (= twice the number of double points) is given by Fulton's formula by

$$
h^{*} h_{*}\left[X^{\prime}\right]-c_{2}(N)
$$

where $N$ is the 'virtual bundle'

$$
N=h^{*} T_{I \times B_{1}}-T_{X^{\prime}}=g^{*}\left(T_{I}\right)-\left(T_{X^{\prime}}-\pi^{*} T_{B_{1}}\right) .
$$

To compute the first term in (30), note that $A^{2}\left(I \times B_{1}\right)$ is generated by the classes

$$
H_{1}^{1}, H_{2}^{2}, \beta H^{1}, \beta H^{2}
$$

where $H_{i}$ are the pullbacks of the hyperplane classes via the two projections of $I$ to $\mathbb{P}^{2}$ and $\beta$ is the class of a point on $B_{1}$. Note that

$$
g^{*} H_{1}=L, g^{*} H_{2}=2 L+K-E
$$

(the latter by (22)), therefore

$$
\begin{gathered}
g_{*} X^{\prime} \cdot \beta \cdot H_{1}=d, g_{*} X^{\prime} \cdot \beta \cdot H_{2}=2 d-2, \\
g_{*} X^{\prime} \cdot H_{1}^{2}=2 N_{d}, g_{*} X^{\prime} \cdot H_{2}^{2}=: 2 M_{d},
\end{gathered}
$$

where, by (22) and (14),

$$
2 M_{d}=(2 L+K-E)_{X^{\prime}}^{2}=2\left(4 N_{d}+4 L \cdot X K-2 N_{d}^{\mathrm{red}}-\kappa_{d}\right)=2\left(N_{d}+L \cdot X K\right) .
$$

Consequently, we have

$$
h_{*}\left[X^{\prime}\right]=(2 d-2) H_{1}^{2}+d H_{2}^{2}+2 M_{d} H_{1} \beta+2 N_{d} H_{2} \beta
$$


from which it is immediate that

$$
h_{*} h^{*}\left[X^{\prime}\right]=(12 d-8) N_{d}+4 d L K
$$

(where we set $L K=L \cdot{ }_{X} K$ ).

The computation of the second term in (30) is straightforward, and can be shortened somewhat by realizing $N$ as the virtual bundle (or 'K-theory element') $g^{*} T_{I}-T_{X^{\prime} / B_{1}}$ where $T_{X^{\prime} / B_{1}}$ is the virtual bundle $T_{X^{\prime}}-\pi^{*} T_{B_{1}}$, and also coincides with the torsion-free sheaf $\omega_{X^{\prime} / B_{1}}^{-1}(-\sigma)$, where $\sigma$ is the set of critical point of $\pi$, whose number is $2 N_{d}^{\mathrm{red}}+2 \kappa_{d}$. From this it is immediate that

$$
c\left(T_{X / B}\right)=1+\left[\omega_{X^{\prime} / B_{1}}^{-1}\right]+[\sigma]=1-K-E_{\kappa}+[\sigma] .
$$

The Chern classes of $I$ can be computed in many ways, for example realizing it as a divisor of type $(1,1)$ on $\mathbb{P}^{2} \times \mathbb{P}^{2}$, and we get

$$
c_{1}\left(T_{I}\right)=2\left(H_{1}+H_{2}\right), c_{2}\left(T_{I}\right)=6 H_{1} H_{2} .
$$

Then putting (35) and (36) together we get

$$
\begin{aligned}
c_{2}(N) & =6 H_{1} H_{2}+2\left(H_{1}+H_{2}\right) \cdot X^{\prime}\left(K+E_{\kappa}\right)+\left(K+E_{\kappa}\right)^{2}+[\sigma] \\
& =24 N_{d}+24 L K-8 N_{d}^{\mathrm{red}}
\end{aligned}
$$

and combining this with (34) we get (29) again.

It remains to work out the contributions from the extraneous double points of $g$, beginning with those related to cusps. Consider a component $E_{\kappa, 1}$ of $E_{\kappa}$ and its unique intersection $q$ with the other fibre component $F$. Locally near $b(q), f$ has the form (20). Therefore near $q$, we have local coordinates

$$
u, v:=\frac{t}{u}
$$

and $g$ is given by

$$
\left(f, \frac{d y}{d x}\right)=\left(u\left(u--\frac{2}{3} v\right),(u-v) u^{2}, \frac{3}{2} u-v\right)
$$

while $h$ is given by

$$
\left(f, \frac{d y}{d x}, t\right)=\left(u^{2}-\frac{2}{3} t,(u-v) u^{2}, \frac{3}{2} u-v, u v\right) .
$$

It is clear from this that the image of the differential $d g_{q}$ is 1 dimensional, equal to $T M:=T_{g(q)} M$, where $M$ is a fibre of $\phi$ (that is, the $\mathbb{P}^{1}$ of lines through $f(q)$ ), and that there is a unique length-2 subscheme $z$ supported at $q$ (viz. the one contained in the line $v=\frac{2}{3} u$ ) that is a double point for $g$ and $h$. 
Moreover, $z$ clearly coincides with the scheme-theoretic fibre of $h$ over $h(q)$, and it is elementary from (39) that $h$ is scheme-theoretically injective over any point other than $h(q)$, hence $z$ counts once as a double point of $h$, so twice as ordered double point of $h$. Recalling that in the 2:1 cover $B_{1} \rightarrow B$ there are $2 \kappa_{d}$ 'cuspidal values', we get a total of $4 \kappa_{d}$ to be subtracted from (29) (recall that $\kappa_{d}$ is given by (14)).

It remains to compute the contribution to the number of virtual double points coming from a component $E_{\sigma}^{1}$ of $E_{\sigma}$. This can be done either using relative double points and the space $Y$ above, or working directly with double points of $h$. We will work out the latter approach, the former being rather similar. Now using local coordinates as in the proof of Lemma 3 on $X, X_{B_{1}}$ is locally embedded in $x, y, s$ space with the equation

$$
x y=s^{2}
$$

the map to $B_{1}$ given by $\pi(x, y, s)=s$. After the blowup we get, near one of the two intersection points of $E_{\sigma}^{1}$ with the other fibre components, local coordinates $x, z$ on $X^{\prime}$ with

$$
s=x z, y=z s=x z^{2}
$$

and in terms of $x, z$ the lift of $f$ is given by $\left(x, x z^{2}\right)$. To work out $g$, note that the relative dualizing sheaf $\omega_{X^{\prime} / B_{1}}$ is locally generated by

$$
\eta=\frac{d x}{x}=-\frac{d z}{z}
$$

so

$$
d x=x \eta, d y=z^{2} d x+2 z x d z=-z^{2} x \eta,
$$

thus $g$ is given by

$$
\left(f, \frac{d y}{d x}\right)=\left(x, x z^{2},-z^{2}\right)
$$

and $h$ by

$$
\left(x, x z^{2},-z^{2}, x z\right) \text {. }
$$

From this it is elementary that $h$ is scheme-theoretically injective off the locus $x=0$ (i.e. $E_{\sigma}^{1}$ ) and, identifying the target locally with 4 -space with coordinates $(w$.$) , the image of h$ is defined by the equations

$$
w_{2}=w_{1} w_{3}, w_{4}^{2}=w_{1}^{2} w_{3},
$$

hence has 2 branches with distinct tangent planes on the $w_{3}$-axis off the origin; moreover $h$ maps $E_{\sigma}^{1} 2: 1$ to this $w_{3}$-axis, which is just a fibre $M$ of $I$ over $\mathbb{P}^{2}$, that is, the $\mathbb{P}^{1}$ of lines through the appropriate node in $\mathbb{P}^{2}$ and $\left.h\right|_{E_{\kappa}^{1}}$ is branched precisely at the two intersection points of $E_{\sigma}^{1}$ with the other fibre components. 
This gives rise to a 1-dimensional double locus component of $g$ and $h$, day $D$, which is a divisor of type $(1,1)$ on $E_{\sigma}^{1} \times E_{\sigma}^{1}$ Now standard double-point theory computes the double locus of $h$ as follows: let $Z$ denote the blow-up of $X^{\prime} \times X^{\prime}$ in its diagonal, with exceptional divisor $E_{Z}$, and let

$$
h_{2}: Z \rightarrow\left(I \times B_{1}\right)^{2}
$$

be the natural map. Then the double-point scheme $\mathfrak{D}$ on $Z$ is a zero-scheme of the rank-4 bundle

$$
h_{2}^{*}\left(A \oplus \mathcal{O}\left(\Delta_{B_{1}}\right)\right)\left(-E_{Z}\right),
$$

and $c_{4}$ of this bundle, pushed down to $X^{\prime}$ is the virtual number (29). Now residual-intersection theory as in $[\mathrm{F}]$ show that the contribution on $D$ to this $c_{4}$ is given by

$$
c_{1}\left(A \oplus \mathcal{O}\left(\Delta_{B_{1}}\right)\left(-E_{Z}\right)\right) . D-c_{1}\left(N_{D / Z}\right) .
$$

Note that $D . \Delta_{B_{1}}=0$ because $D$ maps to a point in $B_{1}^{2}$. Therefore the first term in (40) equals

$$
\text { D. }\left(3 L_{1}+3 L_{2}+K_{1}+K_{2}-E_{1}-E_{2}-4 E_{Z}\right) .
$$

Where $L_{1}, L_{2}$ are the pullbacks of $L$ to $Z$ via the two projections, etc. Now clearly

$$
E_{\sigma}^{1} E=\left(E_{\sigma}^{1}\right)^{2}=-2
$$

and

$$
D \cdot E_{Z}=2
$$

(the 2 branch points), and of course $K_{i} \cdot D=0$ because $K_{X^{\prime}} \cdot E_{\sigma}^{1}=0$ (as blowing up an $A_{1}$ singularity does not change the canonical bundle), so the total is $4-8=-4$. The second term in (40) is, by the adjunction formula, equal to

$$
\operatorname{deg} K_{\mathbb{P}^{1}}-K_{Z} \cdot D=-2-\left(K_{1}+K_{2}\right) \cdot D-E_{Z} \cdot D=-4 .
$$

So the contribution of $D$ is zero. Totalling things up and dividing by 4 , we get the result (18).

REMARK. The analogous computation of the contribution of $D$, carried out on $Y$, yields

$$
c_{1}(A) . D-c_{1}\left(N_{D / Y}\right)
$$

where the first term equals

$$
\text { D. }\left(3 L_{1}+3 L_{2}+K_{1}+K_{2}-E_{1}-E_{2}-3 E_{Z}\right)=-2
$$

and the second term equals

$$
\operatorname{deg} K_{\mathbb{P} 1}-K_{Y} \cdot D=-2
$$

so again we get a zero contribution. 
CHEck. for $d=4$ we use the inputs $N_{d}=620, N_{d}^{\text {red }}=2124, L . K=$ $1564, \kappa_{4}=2304$ to conclude $N_{d}^{\mathrm{tac}}=1296$, a number first computed by Zeuthen [Z], who was also the first to compute $\kappa_{4}$. I am very grateful to Joachim Kock for this and numerous other enlightening references which have led me to correct an error in an earlier statement of Theorem 2 .

Note that the number $M_{d}$ which appeared above is none other than the number of curves in the pencil $B$ which are tangent to a fixed line in $\mathbb{P}^{2}$, so by (32) we conclude

COROLlARY 4. The number of rational curves of degree $d$ in $\mathbb{P}^{2}$ through $3 d-2$ fixed points and tangent to a fixed line is

$$
M_{d}=N_{d}+L K
$$

Note that $L K$ is computed in $\left(12^{\prime}\right)$. As Kock points out, Zeuthen already computed that $M_{4}=2184$.

REMARK. In a previous version of this paper we included a computation of the number of rational plane curves of degree $d$ with a triple point going through $3 d-2$ fixed points. This result is now treated in [R6] with general relative multiple-point techniques, a proof that is very similar to the one we gave originally. Thus it seems best to omit that proof and simply refer to [R6].

\section{REFERENCES}

[CR] M. C. Chang - Z. RAn, Dimension of families of space curves, Comp. Math. 90 (1994), 53-57.

[DH] S. Diaz - J. Harris, Geometry of Severi varieties, Trans. Amer. Math. Soc. 309 (1988) $1-34$.

[F] W. Fulton, "Intersection theory", Springer, 1984.

[FP] W. Fulton - R. PAndharipande, Notes on stable maps and quantum cohomology, In: “Algebraic Geometry”, Proceedings Santa Cruz 1995, D. R. Morrison (ed.), pp. 45-96. Providence, R.I.: Amer. Math. Soc., 1996.

[KP] S. KLeiman - R. Piene, Enumerating singular curves on surfaces, In: "Algebraic geometry", Hirzebruch 70, Contemporary Math. 241 (1999), 209-238, corrections and revision in math.AG/9903192.

[P] R. PANDhARIPANDE, The canonical bundle of $\bar{M}_{d, 0}$ and enumerative geometry, Internat. Math. Res. Notices, 1997, 173-186.

[R1] Z. RAN, Enumerative geometry of singular plane curves, Invent. Math. 97 (1987), 447-465.

[R2] Z. RAN, Bend, break and count, Israel J. Math. 111 (1999), 109-124.

[R3] Z. RAN, Bend, break and count II, Math. Proc. Camb. Phil. Soc. 127 (1999), 7-12.

[R4] Z. RAN, On the variety of rational space curve, Israel J. Math. 122 (2001), 359-370.

[R5] Z. RAN, The degree of the divisor of jumping rational curves, Quart. J. Math. (2001), 1-18.

[R6] Z. RAN, Geometry on nodal curves, (preprint math.AG/0210209). 
[Z] H. Zeuthen, Almindelige Egenskaber ved Systemer af plane Kurver, Vidensk. Selsk. Skr., 5 Række, naturvidenskabelig og mathematisk Afd., Nr. 10, B. IV, København (1873), 286-393.

[Zi] A. ZINGER, Enumeration of 1-nodal rational curves in projective spaces, math.AG 0204236.

Mathematics Department University of California Riverside CA 92521, USA ziv@math.ucr.edu 